\title{
TERAPIA FAMILIAR EN LOS TRASTORNOS DE PERSONALIDAD FAMILY THERAPY IN PERSONALITY DISORDERS
}

\author{
Laura Rodríguez Moya ${ }^{\mathrm{a}}$ Y José Carlos Peláez \\ a Psicóloga Clínica. Centro de Atención Integral a Drogodependientes CAID Sur, de la Comunidad \\ de Madrid.laura_moya@hotmail.com \\ b Médico Psiquiatra. Hospital de Día de Trastornos de la Personalidad. Hospital Clínico San Carlos. Madrid
}

Cómo referenciar este artículo/How to reference this article:

Rodríguez-Moya, L. y Peláez, J. C. (2013). Terapia familiar en los trastornos de personalidad. [Family therapy in personality disorders]. Acción Psicológica, 10(1), 75-84. http://dx.doi.org/10.5944/ap.10.1.7035

\section{Resumen}

La enfermedad mental afecta no solo al paciente sino también a su familia, dificulta la comunicación intrafamiliar, puede generar patologías adicionales en los familiares y todo ello empeorar la evolución del trastorno. Las terapias familiares de tipo psicoeducativo mejoran el pronóstico de la enfermedad y además previenen la aparición de otros trastornos en los familiares. Los elementos más importantes de estas intervenciones son la psicoeducación sobre el trastorno, el re-establecimiento de una relación «sana» entre paciente-familia, el establecimiento de límites, la mejora de la comunicación familiar y el establecimiento de relaciones con la red social extensa, para evitar el aislamiento tanto de la familia como del paciente. Las intervenciones mixtas, individuales y familiares, han demostrado ser muy útiles en el caso de los trastornos de personalidad, en concreto en el Trastorno Límite de Personalidad (TLP).

Palabras clave: Terapia familiar; Psicoeducación; Trastorno de Personalidad; Trastorno límite de la personalidad.
Abstract

Mental illness affects not only the patient but also their families, it can difficult interpersonal communication, can cause additional pathologies inside the family and all this can worsen the evolution of the disorder. Psychoeducational therapies with families improve the prognosis of the disease and furthermore prevent the occurrence of other disorders in relatives. The most important elements of these therapies are: psychoeducation of the disorder, re establishment of a healthy relationship between patient and family, establishment of limits, improvement of family communication, and the establishment of relations with extensive social network to avoid the isolation both family and patient. Mixed therapeutic interventions, individual and family, have shown to be very useful in personality disorders, especially in borderline personality disorder.

Keywords: Family Therapy; Psychoeducation; Personality disorder; Borderline personality disorder. 


\section{Impacto de la patología en el entorno familiar}

El proceso de enfermar no es algo individual, sino que incluye y afecta al contexto familiar del paciente.

Ante la aparición del trastorno, la familia puede reaccionar de diversas formas: resistirse a aceptar que algo no va bien y negar el trastorno, o bien desarrollar conductas de hipervigilancia, sobreprotección y/o codependencia sobre el paciente. Además, el trastorno puede generar otro tipo de patologías en la familia: desgaste emocional, cuadros depresivos, problemas de pareja, violencia doméstica, etc., que interfieran en el diagnóstico inicial. Por otro lado, diversos estudios también destacan la relación entre el contexto familiar y experiencias tempranas de la infancia (traumas, negligencia en cuidados o patología previa de los progenitores) y el desarrollo posterior de una enfermedad mental (Linares, 2007).

Desde una perspectiva sistémica se entiende que la familia juega un importante papel no solo en el proceso generador de la enfermedad, sino también en el proceso de rehabilitación (Olabarría, 2009).

Se ha constatado que el trabajo terapéutico con las familias influye en un mejor pronóstico de la enfermedad mental y además previene la aparición de trastornos adicionales en los familiares (Gunderson, 2002). Las intervenciones mixtas, individuales y familiares, han demostrado ser muy útiles en el caso de los trastornos de personalidad, en concreto en el Trastorno Límite de Personalidad (TLP) (Díaz Curiel, 2001).

El trabajo psicoterapéutico con estas familias debe ir orientado a proporcionar información sobre la enfermedad, modificar ciertas respuestas familiares, proporcionar apoyo en situaciones de crisis o de dificultad especial, competencias para mejorar la comunicación con el familiar afectado, y aprendizaje de habilidades de resolución de problemas y afrontamiento de conflictos, que la continuada convivencia con la enfermedad puede generar. Así como trabajar con las posibles patologías pre- vias que presenten otros miembros del sistema familiar y la historia de traumas previos.

\section{Breve revisión histórica de la intervención familiar en los trastornos de personalidad}

Las primeras investigaciones en el marco de la terapia familiar y la enfermedad mental tienen su origen en los años 50 en EEUU, con los descubrimientos de G. Bateson y el Grupo de Palo Alto, dentro del ámbito de la esquizofrenia. Estos autores defienden la importancia de descentralizar el foco de tratamiento de los síntomas individuales y desplazarlo hacia el contexto inmediato con el que el sujeto se desarrolla e interactúa. Para G. Bateson (1984) la mente humana es eminentemente relacional, y la identidad se desarrolla a través de las interacciones que los individuos establecen con su entorno.

De la mano de estos primeros estudios en el marco de la esquizofrenia, nacen las primeras intervenciones con familiares de pacientes con trastorno de personalidad, en los años 70 también en EEUU. Éstas se centraron, sobre todo, en el Trastorno Límite de Personalidad (TLP). Un grupo de terapeutas de orientación analítica comenzó a estudiar la conexión entre los acontecimientos de los primeros años de la vida y el desarrollo de la personalidad posterior, bajo la hipótesis de que las experiencias de la infancia y la niñez podían determinar que un individuo se desarrollase o no con predisposición a padecer una enfermedad mental en la vida adulta (E. R. Shapiro, Zinner, R. L. Shapiro y Berkowitz. 1974; Shapiro, 1978a, 1978b, 1982).

El estudio de Masterson y Rinsley (1975) marcó un antes y un después en este línea de trabajo. Estos autores plantearon que la familia podía estar en la génesis y/o el mantenimiento de la patología, de manera que, para corregir la psicopatología límite había que corregir a la familia de estos pacientes. A partir de estas primeras publicaciones se desarrollan diversas líneas de investigación centradas en el estudio de la personalidad y su relación con el contexto familiar (E. R. Shapiro, Zinner, R. L. 
Shapiro y Berkowitz. 1974; Shapiro, 1978a, $1978 b, 1982)$. Se empezó a hablar de la «sobreimplicación parental», referido a un estilo educativo parental sobreprotector y sobreimplicado que podía generar dependencia y miedo al abandono en los hijos, e influir así mismo en el desarrollo de psicopatología a lo largo de su vida adulta.

En la década de los 80, algunos investigadores observaron que las familias de este tipo de pacientes podían presentar otro tipo de disfunciones de la comunicación. (Gunderson y Zanarini, 1989). Se empezó a hablar de «negligencia familiar» para referirse a familias que habían prestado muy poca atención y ayuda a los pacientes en las primeras etapas de su vida (Frank, y Paris, 1981; Gunderson, Kerr, y Englund, 1980; Soloff y Millward, 1983); y se observó además una relación significativa entre este tipo de relación y la presencia de experiencias traumáticas en la infancia de estos pacientes (Gunderson y Sabo, 1993; Links y van Reenkum, 1993, Millon, 1987; Paris, ZweigFrank, y Guzder, 1994a, 1994b). A partir de este momento, el tema central del estudio con familias fue «el trauma» y "la negligencia» y su relación con el desarrollo de trastornos de personalidad en la vida adulta (Gunderson, y Zanarini, 1989). Así mismo, se empezó a investigar la historia vital de estos progenitores y se observó que un número importante presentaban a su vez problemas psiquiátricos como abuso de sustancias, depresión y TLP (Goldman, D’Angelo y DeMaso, 1993; Silverman et al., 1991). A partir de este momento, la investigación se centró en el estudio de "la psicopatología de las figuras parentales» y cómo ésta podía influir en el establecimiento de vínculos de apego disfuncionales con los hijos y en el desarrollo de trastornos de personalidad en la vida adulta.

Inicialmente se pensó que la terapia familiar intensiva con estas familias podía provocar cambios curativos a largo plazo. Pero la complejidad de estas familias hacía cada vez más difícil la intervención sobre ellas por lo que, durante un tiempo, se llegó a pensar que no se podía hacer nada por ellas. Así mismo, las familias se sintieron muy juzgadas por estos últimos planteamientos en los que se les hacía responsables de la enfermedad y tampoco se mostraron partidarias de colaborar en los tratamientos.

Otras investigaciones realizadas de forma paralela con pacientes con esquizofrenia arrojaron algo de luz sobre estos planteamientos. Algunos autores observaron que aquellos pacientes que, después de ser dados de alta, volvían a sus domicilios, recaían más que aquellos que se separaban de sus padres y convivían con otras personas ajenas a sus familias. En concreto presentaban índices de recaída mucho más elevados (50 frente a 14\%) en un periodo de 9 a 12 meses (Goldstein, 1995; Leff, 1989; McFarlane y Dunne, 1991). Se utilizó el término "emoción expresada» (Leff, y Vaughn, 1981) para referirse a un estilo de comunicación en el que los familiares mostraban una elevada sobreimplicación y un elevado número de comentarios hostiles y críticas hacia los enfermos. Se diseñaron modelos de intervención basados en enseñar a las familias en qué consistía la enfermedad y en dar pautas para su manejo en el contexto familiar. Se observó que este tipo de intervenciones "psicoeducativas» con estas familias podían reducir la emoción expresada y en consecuencia disminuir la tasa de recaída de los pacientes de forma significativa (Leff $\mathrm{y}$ Vaughn, 1981)

A partir de este momento, las terapias familiares "psicoeducativas» cobraron un especial protagonismo, no solo en el ámbito de la esquizofrenia, sino también con otros trastornos, en concreto con los trastornos de personalidad. Estudios de eficacia demostraron una superioridad de este modelo frente a las primeras terapias familiares de orientación psicoanalítica (Gabbard, Lazar, Hornberger y Spiegel, 1997). Se planteó que la «emoción expresada» podía constituir un factor de recaída o empeoramiento y que el tratamiento debía de ir dirigido a modificar las dinámicas de comunicación disfuncionales dentro de la familia.

\section{La intervención familiar psicoeducativa}

En la actualidad, las intervenciones familiares de perspectiva "psicoeducativa» son un 
tratamiento complementario al individual y se ha demostrado que favorecen el pronóstico de la enfermedad. (Gunderson, 2002).

\section{Objetivos}

Los objetivos de la terapia familiar psicoeducativa son: 1) psicoeducación sobre el trastorno, de modo que la familia adquiera una adecuada comprensión del mismo; 2) reestablecimiento de una relación «sana» entre paciente-familia (que los familiares puedan verle como persona no solo como enfermo); c) establecimiento de límites y mejora de la comunicación familiar (reducir el nivel de crítica, hostilidad o el manejo disfuncionales de emociones negativas); d) establecimiento de relaciones con la red social extensa, para evitar el aislamiento tanto de la familia como del paciente. (Mosquera, 2007; Mosquera y Ageitos, 2005).

\section{Encuadre}

Si bien la graduación temporal puede variar en función de las características de cada caso individual, podemos establecer algunas directrices generales respecto al encuadre y las fases del tratamiento.

Es frecuente que el primer contacto de estos pacientes y sus familias con profesionales de la salud mental se produzca bien en los servicios de urgencias psiquiátricas, a raíz de una crisis aguda, o bien a través de los centros ambulatorios de salud mental. En este momento, habrá que decidir si el paciente requiere hospitalización de urgencia para estabilizar sus síntomas a nivel clínico o si puede beneficiarse de un tratamiento ambulatorio. En cualquier a de los dos casos, y una vez que el paciente esté estable, la opción más recomendable sería una intervención intensiva en formato de hospital de día.

Inicialmente, las sesiones se establecen con una frecuencia semanal y un encuadre muy estructurado. En estos primeros contactos, se organiza la intervención individual y/o grupal so- bre el paciente $y$ se planifican las intervenciones familiares. Si la tensión emocional o los conflictos entre paciente y familia son importantes, es aconsejable comenzar viendo a la familia por un lado y al paciente por otro, es decir por separado, hasta que se pueda disminuir el nivel de emoción expresada (Gunderson, 2002).

Más adelante, si se ha conseguido una buena alianza tanto con la familia como con el paciente y se han trabajado estas tensiones, se planifican las reuniones conjuntas, con una frecuencia quincenal. Los objetivos de las sesiones conjuntas serán reforzar los logros que el paciente ha ido adquiriendo y el apoyo que la familia le está dando. A partir de este momento, también se empiezan a abordar conflictos específicos que puedan estar surgiendo, asociados a la enfermedad o no, así como patologías previas (Gunderson, 2002).

\section{Alianza terapéutica}

El establecimiento de la alianza terapéutica en la terapia de familia representa un reto no solo por el número de personas involucradas, sino también por la complejidad y diversidad (a veces contradicción) de las motivaciones y expectativas sobre la terapia que tienen los diversos miembros de la familia. El terapeuta debe ser capaz de mostrar receptividad ante las diferentes preocupaciones de todos los miembros de la familia, y crear un contexto seguro y de confianza para todos ellos (Shapiro, 1974), de forma que la terapia sea experimentada por cada miembro de la familia como «nuestra terapia» y no como "tenemos que venir a la terapia de nuestro hijo». (Escudero, 2009)

En las primeras fases, es aconsejable que el rol del terapeuta sea más psicoeducativo y directivo. A medida que avanza el tratamiento, su función cambiará y pasará a ser más un «facilitador» de la comunicación entre la familia y el paciente, que un director de la misma. El objetivo es que la familia gane autonomía para que puedan seguir haciendo cambios por sí mismos (Gunderson, 2002). 


\section{Primeros momentos con la familia}

El objetivo principal en estos primeros contactos es explicar a paciente y familiares qué es lo que está pasando, estabilizar al paciente a nivel sintomatológico (decidir si puede hacerse a nivel ambulatorio o bien requiere hospitalización), y emplazarles a iniciar un tratamiento, preferiblemente en hospital de día (Gunderson, 2002).

\section{Terapeuta: ¿Qué le está pasando a esta familia?}

En estos primeros contactos el terapeuta evalúa qué le está pasando a la familia. Los problemas que más frecuentemente encontramos son: problemas de comunicación, dificultades para manejar sentimientos negativos como la ira o la hostilidad ante determinados comportamientos del paciente (ej: autolesiones, consumo de drogas, promiscuidad, etc.), o dificultades para manejar el temor ante la posibilidad de un suicidio (Gunderson, y Lyoo, 1997). El terapeuta rastrea desde cuándo se están produciendo estos problemas, cómo se sienten todos los miembros y qué soluciones ha intentado utilizar la familia hasta ese momento. Este segundo aspecto es especialmente relevante, porque en no pocas ocasiones las soluciones fallidas que la familia ha intentado implementar pueden llegar a convertirse en nuevos problemas añadidos.

En general, los familiares suelen actuar de dos formas (ambas negativas):

a) Sobreprotección al paciente. La familia se desborda ante la problemática del paciente y como forma de manejar, o «evitar», su temor a que algo malo pueda suceder, reaccionan sobreprotegiendo al paciente y estando muy pendientes de él. Controlan de forma estricta sobre todo lo que hace, de manera que el paciente no tiene intimidad ni puede asumir ningún tipo de responsabilidad o autonomía.

b) Negación la enfermedad. Ante el temor al carácter crónico del trastorno o a su propia implicación en el mismo, la familia también puede negar la enfermedad y actuar «como si» no pasase nada, intentando llevar una vida normalizada; sin tener en cuenta que el paciente no puede hacerlo.

\section{Familia: ¿qué le pasa a nuestro hijo/a?}

La familia en estos primeros momentos está sumida en la confusión y el miedo. Necesita información para comprender lo que le pasa al paciente. En su explicación, el terapeuta debe adaptarse al nivel socio-cultural de la familia y adoptar un estilo de comunicación cercano que facilite a la familia la comprensión de lo que sucede. Normalmente, se aconseja utilizar un lenguaje claro y sencillo, huyendo de tecnicismos, evitando la culpabilización y devolviéndoles que se hace cargo de la sobrecarga que está situación debe estar generándoles.

Así mismo, el terapeuta ayuda a la familia a comprender cómo su manera de afrontar la enfermedad y de relacionarse con el paciente (ej.: sobreimplicación, negación, etc.) afecta al curso del tratamiento y que el objetivo de la terapia familiar es cambiar estos aspectos para establecer una relación más "sana» con él. Estos aspectos se abordarán más adelante.

\section{Terapeuta y Familia: ¿Cómo vamos a afrontar esto}

El diagnóstico de un trastorno mental en un miembro de la familia suele desencadenar una "crisis familiar» y una explosión de sentimientos como culpa, miedo, negación etc. (Mosquera, 2007; Mosquera y Ageitos, 2005). Para que la familia se involucre en el tratamiento y establezca una alianza fuerte con nosotros, necesita sentirse apoyada y comprendida, no juzgada. El terapeuta les transmite que les acompañará en todo el proceso y que será su apoyo.

Pero a la vez, hace hincapié en que como terapeuta él también «necesita su ayuda», tera- 
peuta y familia deben de actuar como un equipo, "para favorecer el pronóstico ellos también deben hacer cambios». El terapeuta les explica que no está ahí para juzgarles sino para ayudarles a desarrollar estrategias de afrontamiento más adaptativas frente al problema.

\section{Establecimiento de una alianza: «Somos un equipo»}

El establecimiento de una alianza fuerte y sólida con la familia es requisito indispensable para cualquier intervención familiar. El establecimiento de esta alianza tanto con el paciente como con la familia evitará abandonos prematuros y permitirá manejar las resistencias que puedan influir en el desarrollo de la terapia (Gunderson, 2002).

Podemos encontrarnos con tres tipos de resistencias en la familia:

a) Resistencia al diagnóstico. Enfrentarse a un diagnóstico de enfermedad mental no es fácil, es frecuente que en los primeros momentos las familias se resistan a aceptar la etiqueta de trastorno. Para trabajar la resistencia al diagnóstico, el terapeuta puede utilizar como recursos la bibliografía y la psicoeducación.

b) Resistencia a implicarse en el tratamiento. No es infrecuente encontrarnos que los miembros de la familia se involucran en diferente grado (ej: mayor implicación de la madre y menor del padre...). Si uno de los padres se involucra más que el otro en el tratamiento pueden surgir conflictos importantes, lo más idóneo es lograr que ambos tomen partido en el tratamiento pero, si no es posible, es aconsejable trabajar con el miembro interesado por separado.

c) Resistencia del paciente a que la familia se involucre. En ocasiones es el propio paciente quien puede negarse o mostrar desconfianza ante la intervención familiar. Si el paciente se muestra reacio a que su familia participe en el tratamiento, debemos explorar los motivos de esta negativa, ya que a menudo tiene que ver con el miedo a no ser comprendido, miedo al rechazo, a la soledad, o a la existencia de hechos traumáticos previos relacionados con algún miembro de la familia. El terapeuta puede trabajar estos temores previamente y analizar junto con el paciente los pros y contras de la intervención familiar. (Gunderson, 2002).

\section{Terapia familiar a largo plazo}

Una vez evaluados y delimitados los problemas que presenta la familia, ofrecida la psicoeducación inicial sobre el trastorno y establecida la alianza terapéutica, se comienza el trabajo familiar propiamente dicho:

\section{Mejorar la comunicación familiar «Aprendiendo a hablar el mismo idioma»}

Como hemos comentado anteriormente, en estas familias suele existir una «alta emoción expresada». Los «comentarios críticos» (valoraciones negativas sobre pensamientos, sentimientos o acciones del paciente) y los «comentarios hostiles» (definiciones negativas sobre el paciente, etc.) son frecuentes. Este tipo de comentarios dificultan el proceso de recuperación del paciente e influyen en posibles recaídas. El terapeuta ayuda a la familia a sustituir esta forma de comunicarse por otra que refuerce las cualidades positivas del paciente así como los logros que progresivamente éste vaya alcanzando. (Mosquera, 2007; Mosquera y Ageitos, 2005).

En otras ocasiones, estas familias presentan una elevada "sobreimplicación emocional» $y$ «comportamientos intrusivos». La familia, se siente insegura y necesita proteger al paciente, de modo que intenta controlar todo lo que éste hace. Esto resta autonomía y responsabilidad al paciente en su proceso de cambio. Se pueden llegar a establecer dinámicas muy ambivalentes e inconstantes en las que se alternen periodos en los que al paciente se le trate como un niño y éste se comporte como tal y periodos en los que el paciente reivindique su libertad como adulto y la familia reaccione ante ello con crítica u hostilidad. El terapeuta ayuda a la familia a manejar su inseguridad y a confiar más en la capacidad del paciente para buscar soluciones por sí mismo. 
El terapeuta también asesora a los familiares para que modifiquen la atribución del "rol de enfermo", de modo que puedan comunicarse con «la parte sana» del paciente y hablen con normalidad de otros temas ajenos al trastorno. Según García Badaracco (2006), mirar a un enfermo como «enfermo» es "potencialmente enfermante» y este hecho impide ver una "virtualidad sana» que existe siempre por más enferma que esté una persona. Cuando a un individuo se le diagnostica un trastorno, se genera en la familia «un círculo vicioso enfermante» y toda conducta o pensamiento del sujeto se interpreta en función de la enfermedad. El terapeuta ayuda a la familia a ver al paciente con "otra mirada», una mirada de esperanza que le permita al paciente sentirse comprendido y aceptado. El paciente se siente comprendido cuando «siente» que el otro ha captado que él tiene un "potencial sano» que puede desarrollar (García Badaracco, 2006)

El terapeuta también instruye a los familiares sobre cómo actuar ante la inestabilidad del paciente (cambios bruscos de humor, dicotomía relacional idealización vs. devaluación del otro, etc.). Ante este tipo de situaciones, lejos de asustarse o reaccionar ante las provocaciones del paciente, es aconsejable que la familia mantenga la calma y aprenda a validar los sentimientos que el paciente está verbalizando. Que puedan transmitirle el mensaje de «lo que tú sientes me importa... y estoy aquí».

El terapeuta ayuda a ambos, a familia ya paciente, a crear un entorno estable y seguro que les permita alcanzar una cierta estabilidad emocional a todos. También les enseña a cuidar de sí mismos y protegerse de las emociones de los demás. De esta forma todos son capaces de alcanzar una comunicación más auténtica y más cercana entre ellos (Mosquera, 2007; Mosquera y Ageitos, 2005).

Establecimiento de límites "¿qué puedo esperar y qué no?»

El terapeuta también instruye a la familia a cuidar la congruencia y la coherencia en sus relaciones y en los mensajes que transmiten. Las personas con TLP tienen muchas dificultades para respetar los límites propios y los de los demás. Pero esto también les pasa a algu- nos familiares, que como hemos dicho anteriormente, suelen sobreimplicarse y sobreproteger al paciente.

El terapeuta ayuda a la familia a establecer límites claros, disminuyendo las posibilidades de ambigüedad. Todos los miembros deben saber cuál va a ser la función de cada uno de ellos en las nuevas dinámicas familiares, todos deben saber qué rol van a desempeñar, cómo van a actuar, hasta dónde pueden intervenir, etc. Esto da coherencia y estabilidad a las interacciones y reduce la posibilidad de malos entendidos, tanto en las pautas de comunicación como en los comportamientos de cada miembro. Si esto no es así, los miembros no sabrán muchas veces qué se espera de ellos, cómo reaccionar, qué puede pasar, etc. Paciente y familiares tendrán que estar dispuestos a escuchar la opinión y los sentimientos de la otra persona y aprender a respetarlos (Mosquera, 2007; Mosquera y Ageitos, 2005).

\section{¿Qué hacer ante situaciones de crisis?}

El terapeuta también instruye a la familia sobre cómo actuar ante las amenazas de suicidio, probablemente el grado más extremo de inestabilidad emocional.

Este tipo de amenazas son frecuentes en estos pacientes. Pueden oscilar entre las «llamadas de atención» o «intentos de manipulación» que no tienen una finalidad autolítica y el «deseo real de quitarse la vida». En la mayoría de las ocasiones es difícil determinar cuál es la intencionalidad que se esconde tras la amenaza, por lo que no es aconsejable que la familia cuestione los sentimientos del paciente en ese momento ni juzgue los motivos por lo que quiere suicidarse (o por los que ya lo ha intentado). En esos momentos, es preferible que intente proporcionarle apoyo y comprensión, de nuevo validando su sufrimiento, y le ayude a valorar otras formas alternativas de resolver lo que le está creando ese malestar (Mosquera, 2007; Mosquera y Ageitos, 2005).

\section{¿Por qué cuando el paciente mejora algu- nos familiares empeoran?}

Todos los cambios, aunque sean positivos, requieren de un tiempo de asimilación y de adaptación. Como hemos dicho anteriormente, 
algunos familiares tienen dificultades para ver «la parte sana» del paciente, se han acostumbrado a relacionarse con la "parte enferma» y no son capaces de adaptarse a la mejoría del paciente, siguen comportándose de forma sobreprotectora o crítica con él. Esto puede obstaculizar el progreso terapéutico. El terapeuta señala a los familiares estas dificultades y les ayuda a adaptarse a los cambios que el paciente va experimentando. El proceso de cambio es en paralelo "cambias tú...y cambiamos nosotros».

"Sólo importa el trastorno...la familia se aísla de la sociedad»

Las familias en las que existe una elevada sobreimplicación y sobreprotección tienden a aislarse del entorno social próximo, abandonan actividades que antes realizaban, evitan relaciones previas, etc y solo se centran en el cuidado del enfermo. Esto suele conducir a un incremento del estrés y de la sobrecarga de todos los miembros.

El terapeuta les explica las consecuencias de este tipo de funcionamiento y les anima a que mantengan sus rutinas habituales y no pierdan sus redes sociales extensas. El mantenimiento de vínculo con el exterior fomentará el bienestar de los miembros proporcionando espacios para hablar de temas al margen de la enfermedad. (Mosquera, 2007; Mosquera y Ageitos, 2005).

\section{Manejo de los problemas específicos}

El terapeuta asesora a la familia en la resolución de los problemas específicos que puedan ir surgiendo a lo largo del proceso de cambio. Les ayuda a identificar los problemas específicos, identificar posibles soluciones, tomar decisiones, ponerlas en marcha, analizar las consecuencias e introducir modificaciones, etc. También explorar la existencia de patologías previas en los progenitores o la presencia de traumas previos y delimita el tratamiento específico sobre estos aspectos.

\section{Efectividad de las intervenciones familiares}

Hasta la fecha se han realizado pocos estudios sobre la eficacia de las terapias de familia en el ámbito de los trastornos de personalidad y los datos han sido poco concluyentes. A día de hoy no existe ninguna terapia psicológica que haya mostrado su eficacia y superioridad frente a otras, ni para los trastornos de personalidad en general, ni para los trastornos de personalidad en particular. (Quiroga y Errasti, 2001).

No obstante, algunos elementos que nos permiten constatar la utilidad de las intervenciones familiares son: la mejorar en la capacidad para controlar los impulsos de los pacientes, la disminución de las conductas autoagresivas y suicidas y el descenso en el número de hospitalizaciones.

\section{Conclusiones}

El objetivo final de la intervención, es que el paciente pueda llevar una vida estable, utilizando sus propios recursos personales y el apoyo de la familia. El tratamiento debe ser lo más individualizado posible, no debemos olvidar que aunque existen una serie de características comunes en todos los que padecen la enfermedad, estamos trabajando con "personas» $\mathrm{y}$ «familias» con características diferentes.

La intervención familiar es un elemento indispensable en el proceso de recuperación del paciente. Permite aliviar la tensión de la familia y ayudar al paciente de la forma que necesita. Los familiares son capaces de empezar a comunicarse con él como persona, no como enfermo y le ayudan, sin sobreimplicarse, permitiéndole ganar progresivamente autonomía.

\section{Referencias}

Bateson, G. y Ruesch, J. (1984). Comunicación. La matriz social de la psiquiatría [Communication. The social matrix of psychiatry]. Barcelona, España: Paidós.

Díaz Curiel, J. (2001). Revisión de tratamientos psicoterapéuticos en pacientes con trastorno borderline de personalidad [Review of psychotherapeutic treatments in patients with borderline personality disorder]. Revista de la Asociación Española de Neuropsiquiatría, 78, 51-70. 
Escudero, V. (2009). La creación de la alianza terapéutica en la Terapia Familiar [The creation of the therapeutic alliance in family therapy]. Apuntes de Psicología, 27(2-3), 247-259.

Frank, H. y Paris, J. (1981). Recollections of family experience in borderline patients. Archives of General Psychiatry, 38, 1031-1034.

Gabbard, G. O., Lazar, S. G., Hornberger, J. y Spiegel D. (1997). The economic impact of psychotherapy: a review. American Journal of Psychiatry, 154(2), 147-155.

García Badaracco, J. E. (2006). Virtualidad sana [Healthy virtuality]. (Manuscrito no publicado).

Goldman, S. J., D’Angelo E. J. y DeMaso, D. R. (1993). Psychopatology in the families of children and adolescents with borderline personality disorder. American Journal of Psychiatry, 150, 1832-1835.

Goldstein, M. J. (1995). Psychoeducation and relapse prevention, International Clinical Psychopharmacology, 9(5), 59-69.

Gunderson , J. G., Kerr, J. y Englund, D.W. (1980). The families of borderlines: a comparative study. Archives of General Psychiatry, 37(1), 27-33.

Gunderson, J. G. y Zanarini, M. C. (1989). Pathogenesis of borderline personality disorder. American Psychiatric Press, 8, 25-48.

Gunderson, J. G. y Sabo, A. N. (1993). The phenomenological and conceptual interface between borderline personality disorder and PTSD. American Journal of Psychiatry, 150(1), 19-27.

Gunderson, J. G. y Lyoo, I. K. (1997). Family problems and relationships for adults with borderline personality disorder. Harvard Review of Psychiatry, 4(5), 272-278.

Gunderson, J. G. (2002).Trastorno Límite de la Personalidad. Guía clínica [Borderline Personality Disorder: A Clinical Guide]. Barcelona: Ars XXI de Comunicación.

Leff, J. P. y Vaughn, C. E. (1981). The role of maintenance therapy and relatives' expressed emotion in relapse of schizophrenia: A two year follow up. British Journal of Psychiatry 139, 40-45.

Leff, J. P. (1989). Controversial issues and growing points in research on relatives expressed emotions. International Journal of Social Psychiatry, 35, 133-145.
Linares, J. L. (2007). La personalidad y sus trastornos desde una perspectiva sistémica [Personality and its disorders from a systemic perspective]. Clinica y Salud, 18(3), 381-399.

Links, P. S. y van Reekum, R. (1993). Childhood sexual abuse, parental impairment and the development of borderline personality disorder. Canadian Journal of Psychiatry, 38, 472-474.

Masterson, J. F. y Rinsley, D. B. (1975). The Borderline Syndrome: the role of the mother in the genesis and psychic structure of the borderline personality. International Journal of Psychoanalysis, 56(2),163-177.

McFarlane, W. R. y Dune, E. (1991). Family pychoeducation and multi-family groups in the treatment if schizophrenia. Directions in Psychiatry, 11(20), 2-7.

Millon, T. (1987). On the genesis and prevalence of the borderline personality disorder: a social learning thesis. Journal of Personality Disorders, 1(4), 354-372.

Mosquera, D. y Ageitos, L. (2005). Más allá de lo aparente. Un acercamiento a los comportamientos, pensamientos y actitudes de los familiares de personas con Trastorno Límite de la Personalidad. Manual para profesionales y familiares [Beyond appearances. An approach to the behaviors, thoughts and attitudes of relatives of people with Borderline Personality Disorder. Manual for professionals and family]. Madrid, España: Pléyades.

Mosquera, D. (2007). Trastorno Límite de la Personalidad. Profundizando en el caos [Borderline Personality Disorder. Delving into chaos]. Madrid, España: Pléyades.

Olabarría, B. (2009). Ensanchar las fronteras de nuestra comprensión sistémica acerca del surgimiento y configuración relacional de los trastornos de personalidad [Widening the boundaries of our systemic understanding of the emergence and relational configuration of personality disorders]. Apuntes de Psicología, 27(2-3), 305-320.

Paris, J., Zweig-Frank, H. y Guzder, J. (1994a). Psychological risk factors for borderline personality disorder in female patients. Comprehensive Psychiatry, 35, 301-305.

Paris, J., Zweig-Frank, H. y Guzder, J. (1994b). Risk factors for borderline personality in male out- 
patients. Journal of Nervous and Mental Disease, 182, 375-380.

Quiroga, E. y Errasti, J. M. (2001). Tratamientos psicológicos eficaces para los Trastornos de Personalidad [Effective psychological treatments for personality disorders]. Psichotema, 13(3), 303-405.

Shapiro, E. R., Zinner, J., Shapiro, R. L. y Berkowitz, D. A. (1974). The influence of family experience on borderline personality development. International Review of Psychoanalysis, 2(4), 399-411.

Shapiro, E. R. (1974). Therapist's attitudes and premature termination in family and individual therapy. Journal of Nervous and Mental Disease, 159, 101-107.

Shapiro, E. R. (1978a). The psychodimanics and developmental psychology of the borderline patient: a review of the literatura. American Journal of Psychiatry, 135, 1305-1315.

Shapiro, E. R. (1978b). Research on family dynamics: indications for family and individual treatment in adolescence. Adolescent Psychiatry, 6, 360-376.

Shapiro, E. R. (1982). On curiosity: intrapsychic and interpersonal boundary formation in family life. International Journal of Family Psychiatry, 3, 69-89.

Silverman, J. M., Pinkham, L., Horvath, T. B., Coccaro, E. F., Klar, H., Schear, S.,... y Siever, L. J. (1991). Affective and impulsive personality traits in relatives of borderline patients. American Journal of Psychiatry, 148, 1378-1385.

Soloff, P. H. y Millward, J. W. (1983). Developmental histories of borderline patients. Comprehensive Psychiatry, 24, 574-588. 